\title{
Use of novel oral anticoagulants for the treatment of venous thromboembolism and its considerations in Asian patients
}

This article was published in the following Dove Press journal:

Therapeutics and Clinical Risk Management

7 October 2014

Number of times this article has been viewed

\section{Yun-Jeong Lee}

College of Pharmacy, Dankook University, Cheonan, Republic of Korea
Correspondence: Yun-Jeong Lee College of Pharmacy, Dankook University, Cheonan 330-7I4,

Republic of Korea

$\mathrm{Tel}+824 \mid 550$ I445

Fax +82 4I 5597899

Email yunlee@dankook.ac.kr
Abstract: Parenteral anticoagulation followed by warfarin has been conventionally used for the treatment of venous thromboembolism (VTE). However, there are numerous troublesome characteristics of warfarin that prompted the development of novel oral anticoagulants (NOACs) for the treatment of VTE. Asians are reported to be at an increased risk of bleeding with warfarin, and while the reported incidence of VTE in Asians is lower than in Caucasians, the annual rate of VTE in Asia is rising along with the need for better oral anticoagulant options. Recently, several Phase III clinical trials with NOACs for the treatment and prevention of VTE recurrence have been published. For the treatment of VTE, the four NOACs - dabigatran, rivaroxaban, apixaban, and edoxaban - each showed comparable efficacy outcomes while resulting in better safety outcomes when compared with conventional treatment. In these trials, Asian patients had comparable efficacy and safety outcomes as other races, except in the edoxaban trial, in which the Asian subgroup had better safety profiles than other races, although further confirmation is necessary. For secondary prevention, dabigatran was compared with conventional treatment and showed similar efficacy and safety outcomes. When NOACs were compared with placebo for secondary prevention of VTE, they showed superior efficacy and increased bleeding except for apixaban, which showed comparable major bleeding and composite of major and clinically relevant nonmajor bleeding rates as placebo. No significant differences in the outcomes based on race were observed in the Asian subgroups for secondary prevention. Therefore, NOACs can be used with similar efficacy and at least similar or superior safety compared with conventional treatment in the treatment of VTE, and at no increased risk in Asian patients.

Keywords: dabigatran, rivaroxaban, apixaban, edoxaban, secondary prevention, Asia

\section{Introduction}

\section{Venous thromboembolism}

Venous thromboembolism (VTE) is a term that collectively refers to deep vein thrombosis (DVT) and pulmonary embolism (PE), both of which involve formation of a thrombus in the vein. ${ }^{1}$ Presentation of VTE is wide ranging, from asymptomatic DVT to fatal PE if the blood supply to the lungs is severely blocked by the thrombus. ${ }^{2}$ Major risk factors for VTE include surgery, malignancy, medical illness, a prior history of DVT, age $>60$ years, obesity, prolonged travel, immobility, thrombophilia, and pregnancy. ${ }^{2}$ High-risk surgeries such as orthopedic surgery involving total or partial hip arthroplasty, neurosurgery with excision or biopsy of brain tissue, and vascular surgery such as embolectomy or endarterectomy of lower limb artery result in 2-3\% incidence of thromboembolism within 91 days of surgery. ${ }^{3}$ To prevent such complications of surgery, pharmacologic thromboprophylaxis with anticoagulants, 
antiplatelets, or mechanical prophylaxis are routinely recommended in patients undergoing various surgeries, based on the VTE risk and the risk of bleeding in each patient. ${ }^{4,5}$

The estimated annual incidence of DVT is 48 per 100,000 and PE is 69 per $100,000,{ }^{6}$ but this incidence is known to vary by race and ethnicity. Comparison of the VTE incidence in different ethnicities indicated that the annual rate of diagnosis for VTE per 100,000 was 101.7 in Europeans versus 25.3 in Asians. ${ }^{7}$ This incidence also differs among Asian ethnicities, with the VTE incidence per 100,000 in various Asian countries reported as follows: South Korea 14, Taiwan 17, Hong Kong 17-21, and Singapore 57. ${ }^{8}$ However, it is speculated that the actual incidence of VTE in Asians may not be markedly lower than in Caucasians, as Asians are also subject to many of the major nongenetic risk factors for VTE as Caucasians, annual rates of VTE in Asia are rising quickly, and the difference in rates between Asians and Caucasians are in part a result of limited access to health care resources in some Asian countries. ${ }^{8}$

Occurrence of VTE can result in increased risk of recurrent VTE, death related to $\mathrm{PE}$, or other complications such as chronic postthrombotic syndrome and pulmonary hypertension. ${ }^{5,9}$ The annual incidence of recurrent VTE in Japan is reported to be $3.6 \% .{ }^{9}$ The 30 -day case fatality rate after a VTE is as high as $10.6 \% .^{10}$ In addition, increased mortality was observed in patients with a longer delay in the availability of diagnostic instruments for VTE diagnosis, as reported in a nationwide Italian survey. ${ }^{11}$

Treatment of VTE is conventionally done with anticoagulants - parenteral anticoagulant followed by warfarin although in cases of hemodynamically unstable PE patients, use of thrombolysis is considered as first line. ${ }^{1,12}$ In acute PE, right ventricular dysfunction may be used as a biomarker to assess the risk of death and major adverse events, and to guide initial treatment. ${ }^{13,14}$ In this article, the review is focused on the treatment and recurrence of VTE with anticoagulants.

\section{Vitamin $\mathrm{K}$ antagonists}

Vitamin K antagonists (VKAs), discovered in the 1940s, used to be the first and only class of oral anticoagulants available. Warfarin is the most widely used type of VKA and it exerts action by inhibiting the synthesis of coagulation factors II, VII, IX, and X by depleting the functional vitamin $\mathrm{K}$ reserve from competitively inhibiting the vitamin $\mathrm{K}$ epoxide reductase complex subunit $1 .{ }^{15}$ Although warfarin has a long history of clinical experience, its use can be troublesome to patients due to several characteristics, such as 1) its potential to interact with other drugs or vitamin $\mathrm{K}$-containing products, 2) its narrow therapeutic index requiring frequent monitoring of international normalized ratio (INR), and 3) its slow onset of action, with a mean half-life of 40 hours, requiring up to 5-7 days for full effect. Warfarin has been reported to have increased sensitivity in an Asian population, and a few studies have suggested that the traditional INR goal of two to three may not be optimal due to increased bleeding tendency in Asians, and have suggested a lower INR goal in an Asian population. ${ }^{16,17}$ For these reasons, there has been a need for novel oral anticoagulants (NOACs) that could be used with better patient acceptance.

\section{Novel oral anticoagulants}

In efforts to meet the unmet needs of the conventional oral anticoagulants, NOACs have been developed. There are currently two new classes of NOACs available: the direct thrombin inhibitor dabigatran and direct factor $\mathrm{Xa}$ inhibitors rivaroxaban, apixaban, and edoxaban. Unlike warfarin, which inhibits multiple steps of the coagulation cascade, the NOACs directly inhibit a single step in the cascade. Dabigatran is orally administered as dabigatran etexilate, which is a prodrug converted by esterases in vivo to active metabolite, dabigatran. Dabigatran binds directly to the active site of thrombin (factor IIa) and exerts its anticoagulant effects by competitive inhibition of thrombin. ${ }^{18}$ The factor Xa inhibitors halt the synthesis of thrombin by inhibition of factor Xa, an enzyme responsible for the conversion of prothrombin (factor II) to thrombin. Compared with warfarin, NOACs have fewer drug and food interactions, more predictable pharmacokinetics and pharmacodynamics requiring less monitoring, and faster onset of action. ${ }^{19}$ However, they do have some drawbacks, such as limitations of use in patients with renal impairment and the absence of a direct antidote. ${ }^{20}$

NOACs first gained approval for nonvalvular atrial fibrillation, and since then approval has been sought for treatment of VTE and postoperative venous thromboprophylaxis. Not all NOACs have the indication for treatment of VTE, with one of the main reasons being the recent availability of Phase III clinical trials for treatment of VTE with these agents. NOACs' approval status for treatment of VTE in various parts of the world is listed in Table 1. The US has approved dabigatran, rivaroxaban, and, just recently, in August 2014, apixaban for the treatment and secondary prevention of VTE. In Europe, dabigatran, rivaroxaban, and apixaban have been approved for this indication. However, this approval process is not as fast-paced in Asia; while dabigatran, rivaroxaban and apixaban have gained approval for VTE treatment and secondary prevention in Korea, none of the NOACs has yet to gain approval for VTE treatment. Of note, edoxaban has 
Table I Approval status of the novel oral anticoagulants (NOACs) for treatment and secondary prevention of venous thromboembolism

\begin{tabular}{lllll}
\hline NOACs & US $^{\mathbf{a}}$ & Europe $^{\mathrm{b}}$ & Korea $^{\mathbf{c}}$ & Japan $^{\mathbf{d}}$ \\
\hline Dabigatran & Approved & Approved & Approved & - \\
& $(20 \mid 4 / 04)$ & $(20 \mid 4 / 04)$ & $(20 \mid 4 / 07)$ & \\
\multirow{2}{*}{ Rivaroxaban } & Approved & Approved & Approved & - \\
& $(20 \mid 2 / 1 \mathrm{I})$ & $(20 \mid \mathrm{I} / 09)$ & $(20 \mid 2 / 06)$ & \\
\multirow{2}{*}{ Apixaban } & Approved & Approved & Approved & - \\
& $(20 \mid 4 / 08)$ & $(20 \mid 4 / 06)$ & $(20 \mid 4 / 09)$ & \\
Edoxaban & - & - & - & - \\
\hline
\end{tabular}

Notes: Dash(-) represents that the drug has not yet been approved for the indication as of 2014/09; afrom US Food and Drug Administration (www.accessdata. fda.gov/scripts/cder/drugsatfda/); b brom European Medicines Agency (www.ema. europa.eu/); cfrom Korean Ministry of Food and Drug Safety (http://ezdrug.mfds, go.kr); ; ${ }^{d}$ fom Pharmaceuticals and Medical Devices Agency, Japan (http://www. pmda.go.jp/english/service/package.html).

yet to be approved or marketed anywhere outside of Japan; its only approved indication in Japan is for the venous thromboprophylaxis in patients undergoing major orthopedic surgery. No article to date has evaluated the four NOACs for the treatment of VTE with a focus on Asian patients. An evaluation of the NOACs for VTE treatment specifically in Asian patients is necessary prior to their wide availability for use in clinical practice.

\section{VTE treatment guidelines}

There are several published guidelines to direct the treatment of VTE in clinical practice. One of the most widely used guidelines is published by the American College of Chest Physicians (ACCP), last updated in February 2012, which suggested treatment with VKA over low molecular weight heparin (LMWH) after the initial therapy with parenteral anticoagulation for the treatment of VTE. ${ }^{1}$ For those who are not treated with VKA, they suggest LMWH over dabigatran or rivaroxaban. The recommended duration of treatment for unprovoked DVT or PE was at least 3 months, followed by evaluation of the risk-benefit ratio to determine the need for an extended therapy. The guideline explained that it did not recommend the NOACs as one of the first choices, since at the time of the guideline preparation, postmarketing studies of safety for NOACs were not available.

Although there are national guidelines regarding prevention and/or treatment of VTE in Asian countries, ${ }^{21-25}$ they are not ideal, as they generally follow the ACCP guidelines rather than risk assessment based on local risk factors. ${ }^{8}$ Additionally, some guidelines only focus on the venous thromboprophylaxis rather than treatment of VTE, and the guidelines in general are not up to date to be able to guide the use of NOACs for treatment of VTE.

Currently published Phase III studies for NOACs in the treatment of VTE are largely divided into two types of studies: treatment of VTE and treatment to prevent recurrence of VTE (secondary prevention). Review of these clinical studies will be conducted in separate sections of this article.

\section{Treatment of VTE}

There are a total of six Phase III trials for treatment of VTE that compared the efficacy and safety of NOAC with the conventional anticoagulation therapy (Table 2) ${ }^{26-31}$ There was a notable distinction between the intervention approaches with the NOACs among trials; dabigatran and edoxaban trials required an initial use of a parenteral anticoagulant (either heparin or LMWH), whereas rivaroxaban and apixaban were used as monotherapy without the use of a parenteral agent. The duration of the interventions varied among the trials, which ranged from 3 to 12 months.

\section{Efficacy}

The primary efficacy end points of trials were recurrent symptomatic VTE, or composite of recurrent symptomatic VTE, and death related to VTE (Table 2). In all trials, comparison of each NOAC to conventional treatment resulted in noninferiority of the primary outcome, but none showed superiority over conventional treatment (Table 3). No significant difference in the secondary efficacy end points was reported in any of the trials comparing the NOACs with the conventional treatment.

\section{Safety}

The primary safety end points of trials were major bleeding, or composite of major and clinically relevant nonmajor bleeding (Table 2). Compared with conventional treatment, apixaban was the only drug that showed significantly less bleeding compared with the conventional treatment in both major bleeding $(0.6 \%$ vs $1.8 \%$, hazard ratio [HR] $0.31,95 \%$ confidence interval [CI] 0.17-0.55) and composite of major and clinically relevant nonmajor bleeding (4.3\% vs $9.7 \%$, HR 0.44, 95\% CI 0.36-0.55) (Table 3). Reduction of major or clinically relevant nonmajor bleeding compared with conventional treatment was significant in dabigatran (RECOVER 5.6\% vs $8.8 \%$, HR 0.63 , 95\% CI 0.47-0.84; RECOVER II $5.0 \%$ vs $7.9 \%$, HR $0.62,95 \%$ CI $0.45-0.84$ ) and edoxaban ( $8.5 \%$ vs $10.3 \%$, HR $0.81,95 \%$ CI $0.71-0.94)$, but not significant for major bleeding alone. The pooled analysis of two trials of rivaroxaban showed significant reduction in major bleeding compared with conventional treatment $(1.0 \%$ vs $1.7 \%$, HR $0.54,95 \%$ CI $0.37-0.79$ ), but did not result in significant difference in composite of major and clinically relevant nonmajor bleeding. ${ }^{32}$ 


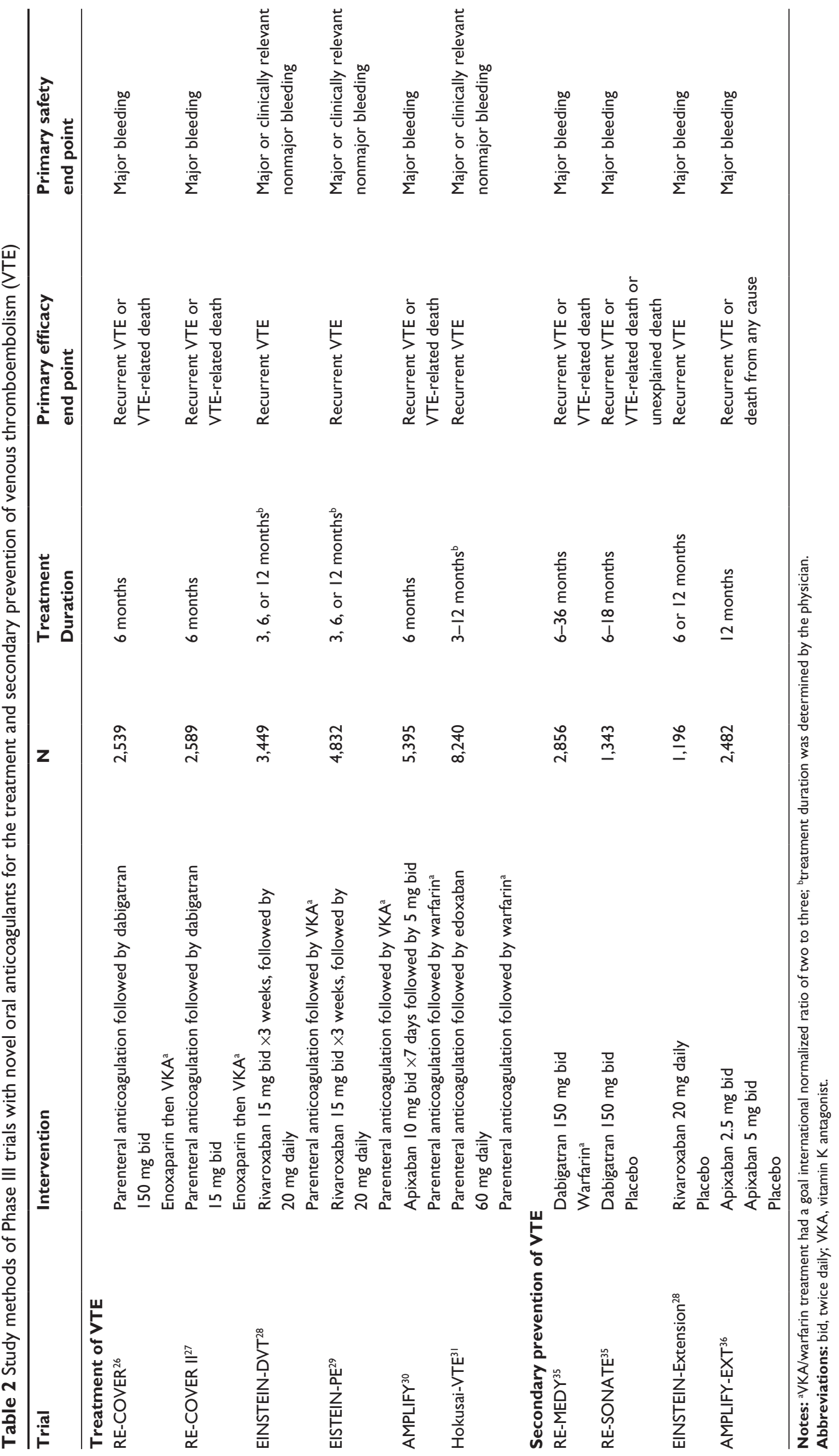




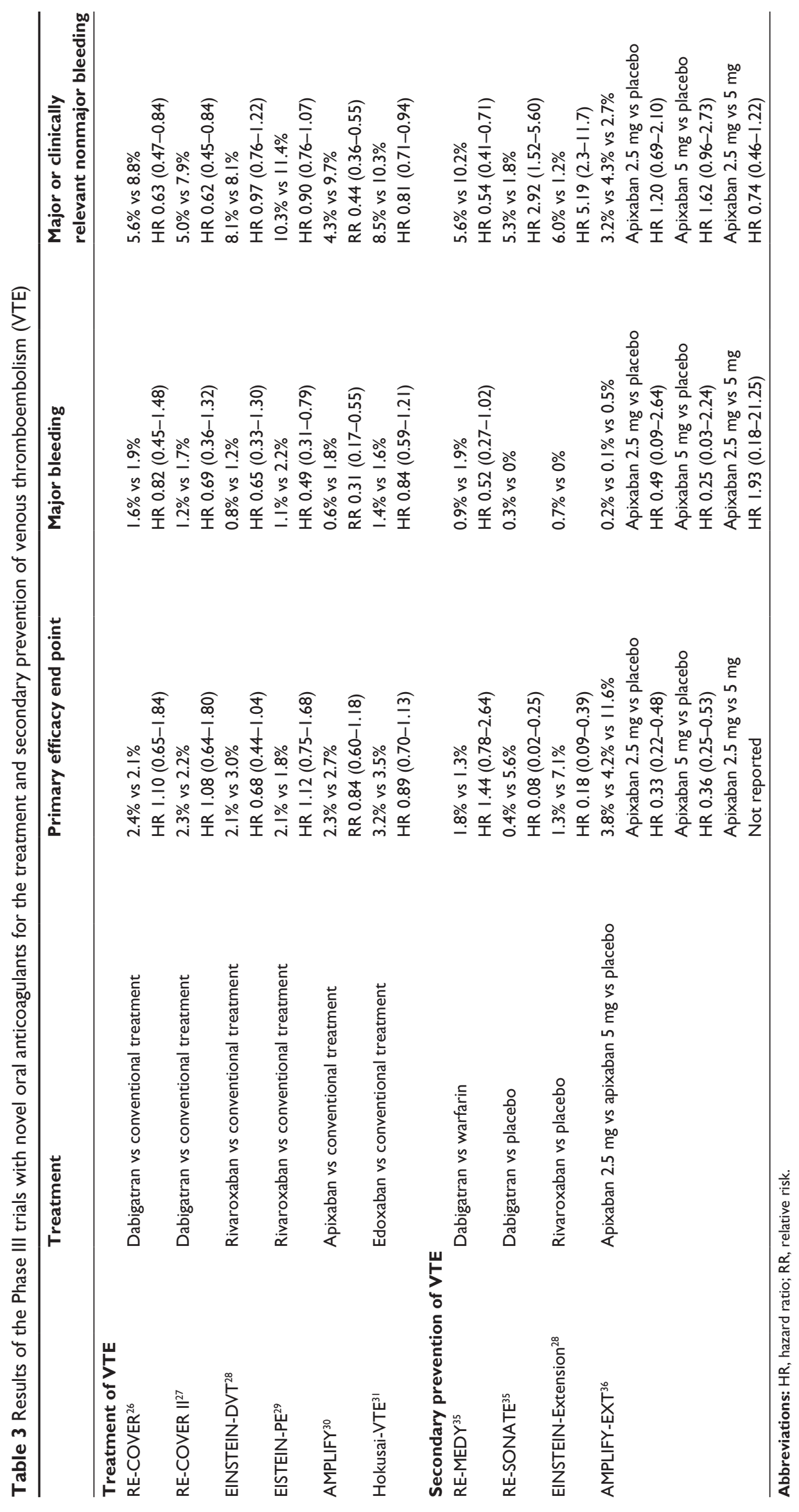




\section{Asian subgroup}

Numbers of Asian patients included in each trial, and Asian patients' primary efficacy and safety outcomes are shown in Table 4. For dabigatran, significantly higher portions of Asian patients were included in the RE-COVER II trial $(20.9 \%)$ than in the RE-COVER trial $(2.6 \%)$, and a pooled subgroup analysis has been performed for the primary efficacy outcome. ${ }^{27}$ Although a higher percentage of Asian patients on dabigatran (2.4\%) had an event compared with conventional treatment (1.0\%), it was not statistically significant, and the $P$-value for interaction for race was $P=0.44$ (Table 4 ). Analysis for safety outcome was not reported.

Primary efficacy and safety end points were analyzed by race for rivaroxaban trials. In both EINSTEIN-DVT and EINSTEIN-PE, Asian subgroups did not significantly benefit from either intervention arms for efficacy or safety. In addition, a subgroup analysis for Chinese patients has been published, which analyzed the results of the 439 Chinese patients included in the EINSTEIN-DVT and EINSTEIN-PE trials. ${ }^{33}$ The overall results for primary efficacy and safety end points seemed to be similar in Chinese patients and the overall population; the primary efficacy end point showed no statistical difference compared with the conventional treatment (3.2\% vs 3.2\%, HR 1.04, 95\% CI 0.36-3.0), and significantly less major bleeding was seen in the rivaroxaban group than in the conventional group ( $0 \%$ vs $2.3 \%)$ in Chinese patients.
However, when examined closely, even though the incidence of the primary safety end point of clinically relevant bleeding in the conventional treatment group was similar in Chinese patients compared with the rest of the population in the study ( $9.2 \%$ vs $10.0 \%)$, the bleeding incidence tended to be lower in Chinese patients compared with the rest of the population in the rivaroxaban group (5.9\% vs $9.4 \%$ ), confirming the favorable safety profile of rivaroxaban for Chinese patients when used for treatment of VTE.

The edoxaban trial Hokusai-VTE included 1,727 (21.0\%) Asians in the study. While the primary efficacy end point did not show any significant difference in the incidences of recurrent VTE among race $(P$-value for interaction $=0.8264)$, the safety end point occurred significantly less in Asians in the edoxaban groups $(8.7 \%)$ compared with Asians in the conventional group (14.5\%) ( $P$-value for interaction for race $=0.0266$ ), and this finding was not observed in any other racial subgroups. This finding suggests a potential for significant safety advantage with use of edoxaban in Asians compared with the conventional group. However, this significance cannot be mechanistically explained by the characteristics of the drug itself, and this finding of enhanced safety in a certain race was not replicated in the edoxaban Phase III atrial fibrillation trial, ENGAGE AF-TIMI 48. ${ }^{34}$ As this finding is based on a subgroup analysis, additional data are needed to confirm that there is a safety advantage of edoxaban in Asians when used for the treatment of VTE.

Table 4 Efficacy and safety outcomes in Asian subgroup

\begin{tabular}{|c|c|c|c|c|}
\hline & Treatment & $\begin{array}{l}\text { Number } \\
\text { of Asians }\end{array}$ & Efficacy & Safety \\
\hline \multicolumn{5}{|l|}{ Treatment of VTE } \\
\hline RE-COVER ${ }^{26}$ & $\begin{array}{l}\text { Dabigatran vs conventional } \\
\text { treatment }\end{array}$ & 65 & $\begin{array}{l}4.0 \% \text { vs } 2.5 \% \\
P \text {-value for interaction }=0.96\end{array}$ & NR \\
\hline RE-COVER $\|^{27}$ & $\begin{array}{l}\text { Dabigatran vs conventional } \\
\text { treatment }\end{array}$ & 537 & $\begin{array}{l}2.4 \% \text { vs } 1.0 \%^{\mathrm{a}} \\
P \text {-value for interaction }=0.44^{\mathrm{a}}\end{array}$ & NR \\
\hline EINSTEIN-DVT ${ }^{28}$ & $\begin{array}{l}\text { Rivaroxaban vs conventional } \\
\text { treatment }\end{array}$ & 494 & $\begin{array}{l}\text { I. } 4 \% \text { vs } 3.8 \% \\
P \text {-value for interaction }>0.05\end{array}$ & $\begin{array}{l}8.4 \% \text { vs } 11.5 \% \\
P \text {-value for interaction }>0.05\end{array}$ \\
\hline EISTEIN-PE ${ }^{29}$ & $\begin{array}{l}\text { Rivaroxaban vs conventional } \\
\text { treatment }\end{array}$ & 287 & $\begin{array}{l}4.1 \% \text { vs } 2.1 \% \\
P \text {-value for interaction }>0.05\end{array}$ & $\begin{array}{l}7.7 \% \text { vs } 9.3 \% \\
P \text {-value for interaction }>0.05\end{array}$ \\
\hline AMPLIFY ${ }^{30}$ & Apixaban vs conventional treatment & NR & NR & NR \\
\hline Hokusai-VTE ${ }^{31}$ & Edoxaban vs conventional treatment & $\mathrm{I}, 727$ & $\begin{array}{l}3.1 \% \text { vs } 3.9 \% \\
P \text {-value for interaction }=0.8264\end{array}$ & $\begin{array}{l}8.7 \% \text { vs } 14.5 \% \\
P \text {-value for interaction }=0.0266\end{array}$ \\
\hline Secondary preventic & of VTE & & & \\
\hline RE-MEDY 35 & Dabigatran vs warfarin & 227 & $\begin{array}{l}0.9 \% \text { vs } 0.9 \% \\
P \text {-value for interaction }=0.978\end{array}$ & NR \\
\hline RE-SONATE ${ }^{35}$ & Dabigatran vs placebo & 118 & $\begin{array}{l}1.4 \% \text { vs } 2.6 \% \\
P \text {-value for interaction }=0.102\end{array}$ & NR \\
\hline EINSTEIN-Extension ${ }^{28}$ & Rivaroxaban vs placebo & NR & NR & NR \\
\hline AMPLIFY-EXT ${ }^{36}$ & $\begin{array}{l}\text { Apixaban } 2.5 \mathrm{mg} \text { vs apixaban } 5 \mathrm{mg} \\
\text { vs placebo }\end{array}$ & NR & NR & NR \\
\hline
\end{tabular}

Notes: All P-values for interaction reported in this trial are on the racial subgroup; ${ }^{a}$ pooled data from RE-COVER and RE-COVER II; ${ }^{\mathrm{b}}$ composite data of Asian and Black patients. Abbreviations: VTE, venous thromboembolism; NR, not reported. 


\section{Secondary prevention of VTE}

For patients with VTE who have received treatment with an anticoagulant for 3-12 months and were in clinical equipose about continuation or cessation of anticoagulant therapy, extended therapy Phase III trials were conducted for secondary prevention of VTE (Table 2). ${ }^{28,35,36}$ Dabigatran trials enrolled patients considered to be at an increased risk of recurrent VTE in the RE-MEDY trial and those considered to be at a decreased risk in the RE-SONATE trial. The RE-MEDY trial compared dabigatran with warfarin with the INR goal of two to three (active control) for 6-36 months, and RE-SONATE compared dabigatran with placebo for 6-18 months. Rivaroxaban and apixaban trials compared the drugs with placebo for 6 or 12 months. No Phase III trial for secondary prevention of VTE was available for edoxaban. The active control trial tested for noninferiority against warfarin, whereas the placebo-controlled trials tested for superiority compared to placebo.

\section{Active control}

Treatment with dabigatran compared with warfarin showed noninferiority in the primary end point of recurrent VTE or death associated with VTE (Table 3), and neither superiority nor inferiority of the groups was observed in all secondary efficacy end points. For safety, there was no significant difference in the rates of major bleeding between dabigatran $(0.9 \%)$ and warfarin (1.9\%) (HR 0.52, 95\% CI 0.27-1.02) (Table 3). However, dabigatran had significantly less major or clinically relevant bleeding (5.6\% vs $10.2 \%$, HR $0.54,95 \%$ CI $0.41-0.71)$ and any bleeding ( $19.4 \%$ vs $26.2 \%$, HR 0.71 , 95\% CI 0.61-0.83) compared with warfarin.

\section{Placebo control}

The primary efficacy end points of the placebo-controlled trials are described in Table 2, which consist of either recurrent VTE or a combination of recurrent VTE and death. As expected, treatment with dabigatran compared with placebo resulted in a significant reduction in the primary end point in the dabigatran group $(0.4 \%$ vs $5.6 \%$, HR $0.08,95 \% \mathrm{CI}$ 0.02-0.25) (Table 3), and this result remained significant up to 12 months after discontinuation of the drug $(6.9 \%$ vs $10.7 \%$, HR $0.61,95 \%$ CI $0.42-0.99$ ). Rivaroxaban also resulted in a significant reduction in primary efficacy end points in comparison with placebo (1.3\% vs $7.1 \%$, HR 0.18 , 95\% CI 0.09-0.39). The same was true for the trial comparing two doses of apixaban versus placebo: $2.5 \mathrm{mg}$ twice daily (3.8\%), $5 \mathrm{mg}$ twice daily (4.2\%), placebo (11.6\%).

An interesting difference among the NOACs was noted in the safety profiles. In RE-SONATE, dabigatran had a 2.92fold increase in major or clinically relevant bleeding versus placebo (95\% CI 1.52-5.60), with numerically more patients in the dabigatran group with major bleeding (two versus none). Rivaroxaban also had a 5.19-fold increase in first major or clinically relevant nonmajor bleeding events versus placebo (HR 5.19, 95\% CI 2.3-11.7), with numerically more patients having major bleeding in the dabigatran group compared with placebo (four versus none, $P=0.11$ ). However, the use of apixaban did not result in increased incidence of the primary safety outcome compared with placebo. Numbers of patients with major bleeding were similar across the groups: apixaban $2.5 \mathrm{mg}$ two patients, apixaban $5 \mathrm{mg}$ one patient, placebo four patients. Moreover, the composite of major and clinically relevant nonmajor bleeding among groups was not significantly

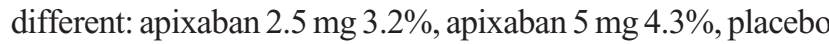
$2.7 \%$ (apixaban $2.5 \mathrm{mg}$ vs placebo HR 1.20, 95\% CI 0.69-2.10, apixaban $5 \mathrm{mg}$ vs placebo HR 1.62, 95\% CI 0.96-2.73, apixaban $2.5 \mathrm{mg}$ vs $5 \mathrm{mg}$ HR $0.74,95 \% \mathrm{CI} 0.46-1.22$ ). This finding is even more astonishing than the previous findings with apixaban trials in patients with atrial fibrillation that reported apixaban to have lower major bleeding rates than warfarin and similar rates of major bleeding compared with aspirin..$^{37,38}$

\section{Asian subgroup}

RE-MEDY and RE-SONATE included 227 and 118 Asian patients in the trials, respectively. However, when subgroup analysis by race was performed, no significant difference in outcomes was observed for race $(P$-value for interaction for both trials $>0.05$ ), indicating that the efficacy would be affected to a similar degree in Asians compared with the rest of the population (Table 4). In rivaroxaban and apixaban trials, a subgroup analysis by race was not reported, although both trials did include Asian patients from various countries.

\section{Consideration of subgroups by weight}

Asians are known to have lower body mass index than nonAsian populations..$^{39}$ Traditionally, the parenteral anticoagulants such as heparin, LMWH, and fondaparinux are dosed based on weight. Weight also plays a role in the dosage requirements of warfarin, where patients with normal weight require significantly lower warfarin doses compared with obese patients. ${ }^{40}$ There have been cases where NOACs have been dose-adjusted by weight or race in previous trials. Apixaban dose was reduced from $5 \mathrm{mg}$ twice daily to $2.5 \mathrm{mg}$ twice daily in two major Phase III trials in patients with atrial fibrillation ${ }^{37,38}$ who had at least two of the following: age $\geq 80$ years, body weight $\leq 60 \mathrm{~kg}$, and serum creatinine level $\geq 1.5 \mathrm{mg} / \mathrm{dL}$, as they were estimated to have higher apixaban exposure. ${ }^{41}$ Variations in dosing requirements for NOACs based on ethnicity have only been performed for rivaroxaban in Japanese patients. In a trial with Japanese 
atrial fibrillation patients treated with rivaroxaban, ${ }^{42}$ it was observed that the Japanese subjects had approximately a $30 \%$ increase in rivaroxaban exposure compared with Caucasian subjects. ${ }^{43,44}$ Consequently, the approved dose of rivaroxaban for atrial fibrillation in Japan is lower (15 $\mathrm{mg}$ daily) than the usual approved dose globally ( $20 \mathrm{mg}$ daily). There is limited information of dabigatran use in patients with body weight $<50 \mathrm{~kg}$, but its product labeling states that based on the clinical and pharmacokinetics data, no dosage adjustment is required, but close clinical surveillance in those patients is suggested. ${ }^{45}$

Fixed doses of NOACs were used in the Phase III trials for VTE treatment and secondary prevention regardless of patients' weight. When examining the subgroup analysis of all Phase III trials for VTE treatment and secondary prevention, no significant differences in outcomes were noted based on subgroups by weight or body mass index. Currently reported data suggest that no weight-based dose adjustment is necessary for the four NOACs in the treatment of VTE.

\section{Future considerations: investigational oral anticoagulants}

There are numerous oral anticoagulants currently under investigation, such as betrixaban (PRT-054021), darexaban (YM150), eribaxaban (PD0348292), letaxaban (TAK-442), and LY517717, which are all factor Xa inhibitors. ${ }^{46}$ However, none of these investigational drugs are currently undergoing trials for treatment or secondary prevention of VTE. Only two of these drugs, betrixaban and darexaban, are currently undergoing or have undergone Phase III trials, respectively, both for venous thromboprophylaxis. ${ }^{47}$

Yet there is an interesting point to be looked out for with regard to one of the investigational drugs, betrixaban. The currently ongoing Phase III betrixaban trial for prevention of VTE is accepting the recruitment of patients with severe renal insufficiency (ie, with a creatinine clearance $[\mathrm{CrCl}]$ of $<30 \mathrm{~mL} / \mathrm{min}$ who are not on dialysis) at a reduced dose, as betrixaban has very low renal excretion (about 5\%), unlike other NOACs. ${ }^{48}$ If the use of a reduced dose of betrixaban results in comparable efficacy without significantly increased bleeding rates, this would become the first of the NOACs with evidence in patients with severe renal impairment. Certainly, further studies will be necessary for betrixaban in the treatment of VTE.

\section{Conclusion and clinical implications}

To date, several Phase III clinical trials for the treatment and secondary prevention of VTE with NOACs are published to guide treatment. The four NOACs - dabigatran, rivaroxaban, apixaban, and edoxaban - with currently reported Phase III data have comparable efficacy in the treatment of VTE compared with the conventional treatment with a parenteral anticoagulant followed by warfarin. None showed superiority over conventional treatment. For safety considerations, apixaban showed superiority over the conventional treatment for both major bleeding and composite of major bleeding and clinically relevant nonmajor bleeding, while dabigatran and edoxaban showed superiority in composite of major and clinically relevant nonmajor bleeding and rivaroxaban showed superiority in major bleeding over conventional treatment. Whereas dabigatran and edoxaban should be used after an initial parenteral anticoagulant, rivaroxaban and apixaban should be administered orally without the use of parenteral agents.

For the secondary prevention of VTE where the anticoagulants were continued 3-12 months after the initial VTE treatment, dabigatran compared with warfarin resulted in similar efficacy and major bleeding rates, and had decreased incidence in composite of major and clinically relevant nonmajor bleeding. When dabigatran, rivaroxaban, and apixaban were compared with placebo, each drug showed superiority in the major efficacy outcomes over placebo. However, apixaban was the only drug that had comparable incidence of major bleeding and composite of major and clinically relevant nonmajor bleeding as placebo, which suggests the distinct safety advantage of apixaban compared with other NOACs. Data on secondary prevention of VTE are not yet available for edoxaban.

No significant difference in the efficacy or safety outcomes were observed specifically for Asian populations in terms of the treatment of VTE or secondary prevention of VTE, with the exception of edoxaban, which had significantly increased safety outcomes in an Asian population compared with other races in the subgroup analysis in the treatment of VTE. Safety was not an issue in patients with lower body weight, and no dosage adjustment seemed necessary based on body weight. Unlike warfarin, current evidence suggests that the Asian population is not at risk of increased sensitivity with the NOACs in the treatment of VTE. Therefore, when considering the use of these NOACs for VTE treatment and/or recurrence, use of any of the studied NOACs can be considered. Based on the currently reported data, it appears that there may be an increased safety advantage of edoxaban in Asians, although this should be further confirmed with additional evidence.

When considering the use of these NOACs for treatment of VTE in clinical practice, the following areas should require further attention. First, the dosing schedule of each NOAC should be considered. While rivaroxaban and edoxaban are dosed once daily, apixaban and dabigatran are dosed twice daily, which could potentially decrease patient adherence in clinical practice and result in negative efficacy outcomes. This is especially important as the NOACs do not require 
monthly monitoring as with warfarin or as was done in the clinical trials. Prior to and during the use of dabigatran or apixaban, patient counseling should be performed extensively to promote patient adherence. Secondly, these agents should be used with caution in patients with renal insufficiency. The trials for both the treatment and secondary prevention of VTE included patients with some degree of renal impairment. However, trials for dabigatran, rivaroxaban, and edoxaban excluded patients with $\mathrm{CrCl}$ of $<30 \mathrm{~mL} / \mathrm{min}$. The apixaban trials excluded patients with $\mathrm{CrCl}<25 \mathrm{~mL} / \mathrm{min}$. No dosage adjustments of drugs were made based on patients' renal function. Thus, warfarin should be considered in patients with severe renal dysfunction $(\mathrm{CrCl}<30 \mathrm{~mL} / \mathrm{min})$. Thirdly, these NOACs have limited data in terms of long-term treatment. When combining the duration of treatment and secondary prevention of VTE trials, dabigatran has up to 3.5 years, rivaroxaban and apixaban has up to 18 months, and edoxaban has up to 12 months of treatment duration. When intending to use these agents for extended duration for VTE, limitation of such data should be noted. Lastly, economic considerations play a big role in clinical practice. When comparing the medication cost of 30-day warfarin with the available NOACs in the US, the NOACs were priced about 17- to 19 -fold higher than warfarin. ${ }^{49}$ However, many other drug-related costs for treatment of VTE should be considered when examining the economic impact of these drugs, such as the routine laboratory costs; costs of hospitalization due to recurrent VTE, bleeding, or death; and consideration of patients' quality of life. There has been a study that evaluated the cost-effectiveness of rivaroxaban compared with enoxaparin plus VKA based on the EINSTEIN trials, which concluded rivaroxaban to be a cost-effective option in treatment of VTE compared with warfarin. ${ }^{50}$ Considerations of the overall factors during evaluation for insurance coverage of the NOACs will be essential for increasing patients' accessibility to these agents.

In conclusion, the clinical trials suggest that NOACs can be used for treatment of VTE with similar efficacy and at least similar or superior safety compared with conventional treatment. For secondary prevention of VTE, dabigatran and rivaroxaban showed superior efficacy and increased bleeding compared with placebo, whereas apixaban demonstrated superior efficacy and similar incidence of bleeding compared with placebo. These NOACs have similar efficacy and do not have increased safety concerns when used in an Asian population.

\section{Acknowledgment}

The present research was conducted by the research fund of Dankook University in 2013.

\section{Disclosure}

The author reports no conflicts of interest in this work.

\section{References}

1. Kearon C, Akl EA, Comerota AJ, et al; American College of Chest Physicians. Antithrombotic therapy for VTE disease: Antithrombotic Therapy and Prevention of Thrombosis, 9th ed: American College of Chest Physicians Evidence-based Clinical Practice Guidelines. Chest. 2012;141(Suppl 2):e419S-e494S.

2. National Clinical Guideline Centre (UK). Venous thromboembolic diseases: the management of venous thromboembolic diseases and the role of thrombophilia testing [webpage on the Internet]. 2012. Available from: http://www.nice.org.uk/guidance/CG144/chapter/introduction. Accessed September 10, 2014.

3. White RH, Zhou H, Romano PS. Incidence of symptomatic venous thromboembolism after different elective or urgent surgical procedures. Thromb Haemost. 2003;90(3):446-455.

4. Gould MK, Garcia DA, Wren SM, et al; American College of Chest Physicians. Prevention of VTE in nonorthopedic surgical patients: Antithrombotic Therapy and Prevention of Thrombosis, 9th ed: American College of Chest Physicians Evidence-based Clinical Practice Guidelines. Chest. 2012;141(Suppl 2):e227S-e277S.

5. Kahn SR, Lim W, Dunn AS, et al; American College of Chest Physicians. Prevention of VTE in nonsurgical patients: Antithrombotic Therapy and Prevention of Thrombosis, 9th ed: American College of Chest Physicians Evidence-based Clinical Practice Guidelines. Chest. 2012;141(Suppl 2):e195S-e226S.

6. Silverstein MD, Heit JA, Mohr DN, Petterson TM, O'Fallon WM, Melton LJ 3rd. Trends in the incidence of deep vein thrombosis and pulmonary embolism: a 25-year population-based study. Arch Intern Med. 1998;158(6):585-593.

7. Liao S, Woulfe T, Hyder S, Merriman E, Simpson D, Chunilal S. Incidence of venous thromboembolism in different ethnic groups: a regional direct comparison study. J Thromb Haemost. 2014;12(2): 214-219.

8. Cohen A, Chiu KM, Park K, et al. Managing venous thromboembolism in Asia: winds of change in the era of new oral anticoagulants. Thromb Res. 2012;130(3):291-301.

9. Nakamura M, Miyata T, Ozeki Y, et al. Current venous thromboembolism management and outcomes in Japan. Circ J. 2014;78(3):708-717.

10. Tagalakis V, Patenaude V, Kahn SR, Suissa S. Incidence of and mortality from venous thromboembolism in a real-world population: the Q-VTE Study Cohort. Am J Med. 2013;126(9):832.e13-e21.

11. de Franciscis S, Agus GB, Bisacci R, et al. Guidelines for venous thromboembolism and clinical practice in Italy: a nationwide survey. Ann Vasc Surg. 2008;22(3):319-327.

12. Torbicki A, Perrier A, Konstantinides S, et al; ESC Committee for Practice Guidelines (CPG). Guidelines on the diagnosis and management of acute pulmonary embolism: the Task Force for the Diagnosis and Management of Acute Pulmonary Embolism of the European Society of Cardiology (ESC). Eur Heart J. 2008;29(18): 2276-2315.

13. Serra R, de Franciscis S. The role of thrombolysis for patients with hemodynamically stable acute pulmonary embolism. Thromb Res. 2014;134(1):7-8.

14. Sanchez O, Trinquart L, Colombet I, et al. Prognostic value of right ventricular dysfunction in patients with haemodynamically stable pulmonary embolism: a systematic review. Eur Heart J. 2008;29(12):1569-1577.

15. Whitlon DS, Sadowski JA, Suttie JW. Mechanism of coumarin action: significance of vitamin K eposide reductase inhibition. Biochemistry. 1978;17(8):1371-1377.

16. Yamaguchi T; Japanese Nonvalvular Atrial Fibrillation-Embolism Secondary Prevention Cooperative Study Group. Optimal intensity of warfarin therapy for secondary prevention of stroke in patients with non-valvular atrial fibrillation: a multicenter, prospective, randomized trial. Stroke. 2000;31(4):817-821. 
17. You JH, Chan FW, Wong RS, Cheng G. Is INR between 2.0 and 3.0 the optimal level for Chinese patients on warfarin therapy for moderate-intensity anticoagulation? Br J Clin Pharmacol. 2005;59(5):582-587.

18. Eisert WG, Hauel N, Stangier J, Wienen W, Clemens A, van Ryn J. Dabigatran: an oral novel potent reversible nonpeptide inhibitor of thrombin. Arterioscler Thromb Vasc Biol. 2010;30(10):1885-1889.

19. Ahrens I, Lip GY, Peter K. New oral anticoagulant drugs in cardiovascular disease. Thromb Haemost. 2010;104(1):49-60.

20. Wang Y, Bajorek B. New oral anticoagulants in practice: pharmacological and practical considerations. Am J Cardiovasc Drugs. 2014; 14(3):175-189.

21. Academy of Medicine, Malaysia, Ministry of Health Malaysia, National Heart Association of Malaysia. Clinical Practice Guidelines: Management of Venous Thromboembolism; 2003. Available from: www. acadmed.org.my/view_file.cfm?fileid=278. Accessed September 10, 2014.

22. Nakamura M. Japanese guidelines for prevention of venous thromboembolism. J Japan Soc Clin Anesth. 2004;24(9):480-487.

23. Parakh R, Kakkar VV, Kakkar AK. Management of venous thromboembolism. J Assoc Physicians India. 2007;55:49-70.

24. Chinese Orthopaedic Association. Prevention of venous thromboembolism after major orthopaedic surgery. Orthop Surg. 2010;2(2):81-85.

25. Bang SM, Jang MJ, Kim KH, et al. Prevention of venous thromboembolism, 2nd edition: Korean Society of Thrombosis and Hemostasis Evidence-based Clinical Practice Guidelines. J Korean Med Sci. 2014;29(2):164-171.

26. Schulman S, Kearon C, Kakkar AK, et al; RE-COVER Study Group. Dabigatran versus warfarin in the treatment of acute venous thromboembolism. N Engl J Med. 2009;361(24):2342-2352.

27. Schulman S, Kakkar AK, Goldhaber SZ, et al; RE-COVER II Trial Investigators. Treatment of acute venous thromboembolism with dabigatran or warfarin and pooled analysis. Circulation. 2014; 129(7):764-772.

28. EINSTEIN Investigators; Bauersachs R, Berkowitz SD, Brenner B, et al. Oral rivaroxaban for symptomatic venous thromboembolism. N Engl J Med. 2010;363(26):2499-2510.

29. EINSTEIN-PE Investigators; Büller HR, Prins MH, Lensin AW, et al. Oral rivaroxaban for the treatment of symptomatic pulmonary embolism. N Engl J Med. 2012;366(14):1287-1297.

30. Agnelli G, Buller HR, Cohen A, et al; AMPLIFY Investigators. Oral apixaban for the treatment of acute venous thromboembolism. $N$ Engl JMed. 2013;369(9):799-808.

31. Hokusai-VTE Investigators, Büller HR, Décousus H, Grosso MA, et al. Edoxaban versus warfarin for the treatment of symptomatic venous thromboembolism. N Engl J Med. 2013;369(15):1406-1415.

32. Prins MH, Lensing AW, Bauersachs R, et al; EINSTEIN Investigators. Oral rivaroxaban versus standard therapy for the treatment of symptomatic venous thromboembolism: a pooled analysis of the EINSTEINDVT and PE randomized studies. Thromb J. 2013;11(1):21.

33. Wang $\mathrm{Y}$, Wang $\mathrm{C}$, Chen $\mathrm{Z}$, et al; Chinese EINSTEIN Investigators. Rivaroxaban for the treatment of symptomatic deep-vein thrombosis and pulmonary embolism in Chinese patients: a subgroup analysis of the EINSTEIN DVT and PE studies. Thromb J. 2013;11(1):25.

34. Giugliano RP, Ruff CT, Braunwald E, et al; ENGAGE AF-TIMI 48 Investigators. Edoxaban versus warfarin in patients with atrial fibrillation. N Engl J Med. 2013;369(22):2093-2104.
35. Schulman S, Kearon C, Kakkar AK, et al; RE-MEDY Trial Investigators; RE-SONATE Trial Investigators. Extended use of dabigatran, warfarin, or placebo in venous thromboembolism. $N$ Engl $J$ Med. 2013;368(8):709-718.

36. Agnelli G, Buller HR, Cohen A, et al; PLIFY-EXT Investigators. Apixaban for extended treatment of venous thromboembolism. N Engl J Med. 2013;368(8):699-708.

37. Connolly SJ, Eikelboom J, Joyner C, et al; AVERROES Steering Committee and Investigators. Apixaban in patients with atrial fibrillation. N Engl J Med. 2011;364(9):806-817.

38. Granger CB, Alexander JH, McMurray JJ, et al; ARISTOTLE Committees and Investigators. Apixaban versus warfarin in patients with atrial fibrillation. $N$ Engl J Med. 2011;365(11):981-992.

39. WHO Expert Consultation. Appropriate body-mass index for Asian populations and its implications for policy and intervention strategies. Lancet. 2004;363(9403):157-163.

40. Wallace JL, Reaves AB, Tolley EA, et al. Comparison of initial warfarin response in obese patients versus non-obese patients. $J$ Thromb Thrombolysis. 2013;36(1):96-101.

41. Lopes RD, Alexander JH, Al-Khatib SM, et al; ARISTOTLE Investigators. Apixaban for Reduction in Stroke and Other Thromboembolic Events in Atrial Fibrillation (ARISTOTLE) Trial: design and rationale. Am Heart J. 2010;159(3):331-339.

42. Hori M, Matsumoto M, Tanahashi N, et al; J-ROCKET AF study investigators. Rivaroxaban vs Warfarin in Japanese Patients with Atrial Fibrillation: the J-ROCKET AF study. Circ J. 2012;76(9): 2104-2111.

43. Tanigawa T, Kaneko M, Hashizume K, et al. Model-based dose selection for phase III rivaroxaban study in Japanese patients with non-valvular atrial fibrillation. Drug Metab Pharmacokinet. 2013;28(1):59-70.

44. Kaneko M, Tanigawa T, Hashizume K, Kajikawa M, Tajiri M, Mueck W. Confirmation of model-based dose selection for a Japanese phase III study of rivaroxaban in non-valvular atrial fibrillation patients. Drug Metab Pharmacokinet. 2013;28(4):321-331.

45. Pradaxa ${ }^{\circledR}$ (dabigatran etexilate). Summary of Product Characteristics. 2013 Jan. Available from: http://www.ema.europa.eu/docs/en_GB/ document_library/EPAR_-_Product_Information/human/000829/ WC500041059.pdf. Accessed September 10, 2014.

46. Broussalis E, Anna W, Trinka E, Mutzenbach S, Killer M. Latest developments in anticoagulant drug discovery. Drug Discov Today. 2014; 19(7):921-935.

47. Sakon M, Nakamura M. Darexaban (YM150) prevents venous thromboembolism in Japanese patients undergoing major abdominal surgery: phase III randomized, mechanical prophylaxis-controlled, open-label study. Thromb Res. 2012;130(3):e52-e59.

48. Cohen AT, Harrington R, Goldhaber SZ, et al. The design and rationale for the Acute Medically Ill Venous Thromboembolism Prevention with Extended Duration Betrixaban (APEX) study. Am Heart $J$. 2014;167(3):335-341.

49. Drugstore.com [homepage on the Internet]. Bellevue: drugstore.com, Inc.; c1999-2014. Available from: http://www.drugstore.com. Accessed September 10, 2014.

50. Lefebvre P, Coleman CI, Bookhart BK, et al. Cost-effectiveness of rivaroxaban compared with enoxaparin plus a vitamin $\mathrm{K}$ antagonist for the treatment of venous thromboembolism. J Med Econ. 2014; 17(1):52-64.
Therapeutics and Clinical Risk Management

\section{Publish your work in this journal}

Therapeutics and Clinical Risk Management is an international, peerreviewed journal of clinical therapeutics and risk management, focusing on concise rapid reporting of clinical studies in all therapeutic areas, outcomes, safety, and programs for the effective, safe, and sustained use of medicines. This journal is indexed on PubMed Central, CAS,

\section{Dovepress}

EMBase, Scopus and the Elsevier Bibliographic databases. The manuscript management system is completely online and includes a very quick and fair peer-review system, which is all easy to use. Visit http://www.dovepress.com/testimonials.php to read real quotes from published authors. 